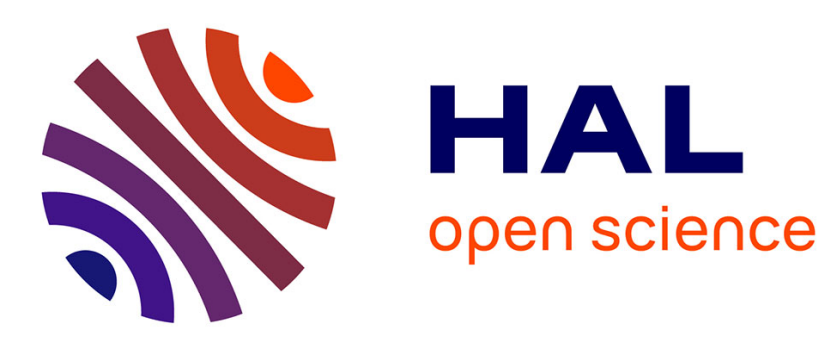

\title{
A falling cloud of particles at a small but finite Reynolds number
}

Florent Pignatel, Maxime Nicolas, Elisabeth Guazzelli

\section{To cite this version:}

Florent Pignatel, Maxime Nicolas, Elisabeth Guazzelli. A falling cloud of particles at a small but finite Reynolds number. Journal of Fluid Mechanics, 2011, 10.1017/S0022112010005501 . hal-01432973

\section{HAL Id: hal-01432973 \\ https://hal-amu.archives-ouvertes.fr/hal-01432973}

Submitted on 12 Jan 2017

HAL is a multi-disciplinary open access archive for the deposit and dissemination of scientific research documents, whether they are published or not. The documents may come from teaching and research institutions in France or abroad, or from public or private research centers.
L'archive ouverte pluridisciplinaire HAL, est destinée au dépôt et à la diffusion de documents scientifiques de niveau recherche, publiés ou non, émanant des établissements d'enseignement et de recherche français ou étrangers, des laboratoires publics ou privés. 


\title{
A falling cloud of particles at a small but finite Reynolds number
}

\author{
FLORENT PIGNATEL, MAXIME NICOLAS \\ AND ÉLISABETH GUAZZELLI† \\ IUSTI-CNRS UMR 6596, Polytech-Marseille, Aix-Marseille Université (U1), \\ Technopôle de Château-Gombert, 13453 Marseille CEDEX 13, France
}

(Received 7 July 2010; revised 27 September 2010; accepted 14 October 2010)

Through a comparison between experiments and numerical simulations, we have examined the dynamics of a cloud of spheres at a small but finite Reynolds number. The cloud is seen to flatten and to transition into a torus, which further widens and eventually breaks up into droplets. While this behaviour bears some similarity to that observed at zero inertia, the underlying physical mechanisms differ. Moreover, the evolution of the cloud deformation is accelerated as inertia is increased. Two inertial regimes in which macro-scale inertia and micro-scale inertia become successively dominant are clearly identified.

Key words: breakup/coalescence, particle/fluid flow, suspensions

\section{Introduction}

The dispersion of a collection of particles is relevant to many natural phenomena such as pyroclastic flows (i.e. fast-moving currents of hot gas and rock fragments produced by a volcanic eruption), turbidity currents (i.e. sediment-laden flows down a slope usually in lakes and oceans) and the mixing and spreading of pollutants in lakes and oceans. In most of these natural phenomena or industrial applications involving dispersion of particles, the particulate flow is dominated by inertial forces. In this paper, we consider the motion of a cloud of spherical particles settling because of gravity in an otherwise pure liquid when the Reynolds number is no longer zero. In contrast to high-Reynolds-number clouds related to some of the geophysical flows mentioned above (see e.g. Noh \& Fernando 1993; Bush, Thurber \& Blanchette 2003) the focus here is on finite-but-moderate-Reynolds-number clouds, which have been scarcely addressed.

Previous work on the dynamics of sedimenting clouds of particles have been undertaken in conditions where inertia is considered as negligible (Adachi, Kiriyama \& Koshioka 1978; Nitsche \& Batchelor 1997; Machu et al. 2001; Metzger, Nicolas \& Guazzelli 2007; Park et al. 2010). The initially spherical cloud is found to evolve into a torus and eventually to break up into (usually two) secondary droplets which themselves deform into tori in a repeating cascade. The sequence of instability and breakup is found to be insensitive to the initial shape of the cloud and occurs in the complete absence of inertia (Metzger et al. 2007). Faster breakup is observed for clouds of fibres due to the self-motion of the anisotropic particles (Park et al. 2010).

$\dagger$ Email address for correspondence: elisabeth.guazzelli@polytech.univ-mrs.fr 


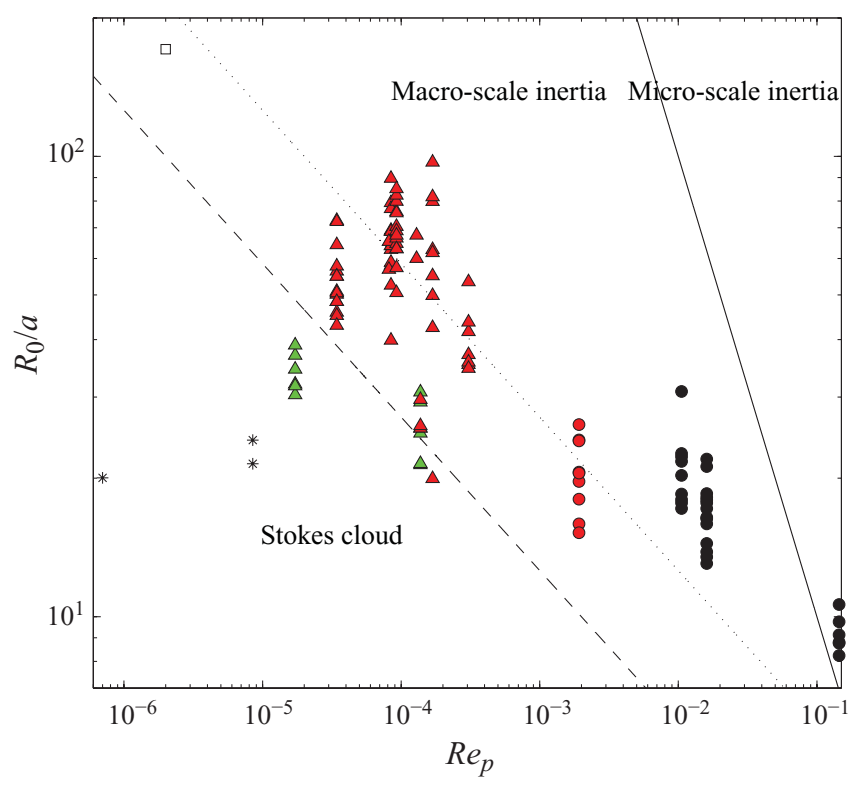

FIGURE 1. The regimes of evolution for a falling cloud of particles as a function of the ratio $R_{0} / a$ of cloud radius to particle radius, particle Reynolds number $R e_{p}=U_{0} a \rho_{f} / \mu$ and volume fraction $\phi$. The dashed and dotted lines $\left(R_{0} / a \sim R e_{p}^{-1 / 3} \phi^{-1 / 3}\right)$ denote transition to a regime dominated by 'macro-scale inertia' for $\phi \approx 50 \%$ and $5 \%$ respectively. The solid line $\left(R_{0} / a \sim R e_{p}^{-1}\right)$ denotes transition to a regime dominated by 'micro-scale inertia'. The symbols are as follows: *, experiments of Metzger et al. (2007) at $\phi \approx 4 \%$ and $20 \%$; $\square$, simulations of Bosse et al. (2005b) for $R e_{c} \sim 1 ; \triangle$ and $O$, present experiments at $\phi \approx 2-10 \%$ and $50 \%$ respectively.

An important feature is that the randomness of the particle velocities causes some particles to depart from the closed toroidal streamlines inside the cloud, to be carried round the boundary and thence downstream in a vertical tail emanating from the rear of the cloud (Nitsche \& Batchelor 1997; Metzger et al. 2007).

A limited number of studies treat the case in which inertia is no longer negligible. Bosse et al. (2005b) investigated numerically the settling dynamics of an initially spherical cloud for low and moderate cloud Reynolds numbers. A Fourier pseudospectral method used to solve the Navier-Stokes equations for the fluid phase was combined with a Lagrangian point-particle tracking for the particle phase. They found that the cloud showed no significant leakage and deformed into a torus that eventually fractured into multiple droplets. The number of droplets was primarily determined by the cloud Reynolds number and the particle distribution inside the initial cloud. Subramanian \& Koch (2008) presented a theoretical analysis of the long-time dynamics of sedimenting clouds, wherein the motion of each particle was governed by the steady Oseen velocity fields due to the other particles inside the cloud. They examined the cases in which particle interactions were dominated by the source or the wake part of this inertial velocity field.

The different regimes of evolution for a sedimenting cloud of particles are presented in figure 1, adapted from figure 1 in Subramanian \& Koch (2008). The first regime corresponds to the 'Stokes cloud', where both the particle Reynolds number $R e_{p}=U_{0} a \rho_{f} / \mu$ and the cloud Reynolds number $R e_{c}=V_{0} R_{0} \rho_{f} / \mu$ are very small. Here we have denoted by $U_{0} \sim\left(\rho_{p}-\rho_{f}\right) a^{2} g / \mu$ the Stokes velocity of an isolated sphere of radius $a$ and density $\rho_{p}$, settling in a fluid of viscosity $\mu$ and density $\rho_{f}$, and 
by $V_{0} \sim \phi\left(\rho_{p}-\rho_{f}\right) R_{0}^{2} g / \mu$ the Stokes velocity of a cloud of radius $R_{0}$ and volume fraction $\phi$, settling in the same fluid, where $g$ is the acceleration of gravity. In the investigations of this regime mentioned earlier (Nitsche \& Batchelor 1997; Machu et al. 2001; Metzger et al. 2007), the interacting particles are treated as point forces, and only the far-field hydrodynamic interactions (Stokeslets) are retained. When inertia is increased, the sedimenting cloud transitions first to a regime dominated by 'macro-scale inertia' when the cloud Reynolds number $\operatorname{Re}_{c}\left(\sim \phi \operatorname{Re}_{p} R_{0}^{3} / a^{3}\right) \sim 1$, i.e. $R_{0} / a \sim R e_{p}^{-1 / 3} \phi^{-1 / 3}$. The numerical simulation of Bosse et al. (2005b) for $R e_{c} \sim 1$ lies in that regime. A second transition to a regime dominated by 'micro-scale inertia' occurs when the inertial length $\ell=a / R e_{p}$ is of the order of the cloud radius $R_{0}$, i.e. $R_{0} / a \sim R e_{p}^{-1}$. This is the regime investigated by Subramanian \& Koch (2008), who considered particles interacting through their steady Oseen velocity fields within the cloud.

The above parameter regimes introduced by Subramanian \& Koch (2008) lead us to discuss the various dimensionless parameters involved in the problem. Following Bosse et al. (2005b), we can identify a total of seven independent physical quantities that uniquely describe the properties of a cloud of particles settling in a fluid under gravity. The fluid is characterised by its viscosity $\mu$ and density $\rho_{f}$, the particles by their radius $a$ and density $\rho_{p}$, the cloud by its radius $R_{0}$ and number $N_{0}$ of particles and, finally, the driving force by the gravitational acceleration $g$. Dimensional analysis indicates that at least four dimensionless parameters have to be specified. An appropriate choice is to consider the particle number $N_{0}$, the dimensionless inertial length $\ell^{*}=\left(a / R_{0}\right) / R e_{p}$, the cloud Reynolds number $R e_{c}$ and the Stokes number $S t=(2 / 9)\left(\rho_{p} / \rho_{f}\right) R e_{p}$. This last dimensionless number is always kept very small and thus does not play a significant role here. It is important to note that the underlying consideration is that long-range interactions dominate the behaviour, and short-range repulsive forces (e.g. contact forces) are largely to be neglected; i.e. the behaviour is believed to hardly depend on the ratio $a^{*}=a / R_{0}$. In this sense, parameters like $N_{0}$ and $\phi=N_{0}\left(a / R_{0}\right)^{3}$ can be considered equivalent. The same holds for the pair $\ell^{*}$ and $R e_{p}=\left(a / R_{0}\right) / \ell^{*}$. The reason for choosing $N_{0}$ and $\ell^{*}$ becomes clear when looking at the Oseenlet simulations detailed in $\S 3$, which describe the interactions between point particles in terms of the inertial length $\ell$ and do not explicitly contain the particle radius $a$. As a consequence, quantities like $R e_{c}, R e_{p}$ and $\phi$ cannot be computed for the simulation results. The conclusion of this discussion is that the appropriate dimensionless parameters to describe the present system are $N_{0}, \ell^{*}$ and $R e_{c}$.

The objective of the present paper is to investigate the dynamics of clouds of particles in regimes in which macro-scale inertia and micro-scale inertia become dominant. This is undertaken by performing experiments that are described in $\S 2$. The experimental data are compared in $\S 4$ with both the simulations of Bosse et al. $(2005 a, b)$ and the Oseenlet simulations inspired by Subramanian \& Koch (2008) which are described in $\S 3$. Conclusions are drawn in $\S 5$.

\section{Experimental techniques}

The experimental techniques were similar to those developed by Metzger et al. (2007). Two glass-walled vessels having dimensions $4 \mathrm{~cm} \times 10 \mathrm{~cm} \times 100 \mathrm{~cm}$ and $20 \mathrm{~cm} \times 20 \mathrm{~cm} \times 100 \mathrm{~cm}$ were used in the experiments. The suspension, i.e. the mixture of particles and fluid, was prepared with a desired volume fraction $\phi$ in a small glass container. Clouds having different initial radius $R_{0}$ were then produced by injecting with a syringe a given volume of this suspension at the top of the vessel filled 


$\begin{array}{clcc}\text { Batch } & \text { Composition } & a(\mu \mathrm{m}) & \rho_{p}\left(\mathrm{~g} \mathrm{~cm}^{-3}\right) \\ \text { A } & \text { Leaded glass } & 51 \pm 4 & 4.085 \pm 0.040 \\ \text { B } & \text { Polymethyl methacrylate } & 76 \pm 10 & 1.178 \pm 0.020 \\ \text { C } & \text { Glass } & 75 \pm 6 & 2.490 \pm 0.010 \\ \text { D } & \text { Glass } & 156 \pm 6 & 2.490 \pm 0.010\end{array}$

TABLE 1. Particle characteristics. The particle density $\rho_{p}$ was measured with a pycnometer and a fluid of measured density $0.823 \mathrm{~g} \mathrm{~cm}^{-3}$. The particle size distributions were determined with a digital imaging system. The mean radius is denoted $a$, and the error corresponds to one standard deviation.

$\begin{array}{cccc}\text { Fluid } & \text { Composition } & \mu(\mathrm{cP}) & \rho_{f}\left(\mathrm{~g} \mathrm{~cm}^{-3}\right) \\ 1 & 10 \% \text { UCON oil }+90 \% \text { water } & 9.2 \pm 0.1 & 1.011 \pm 0.005 \\ 2 & 20 \% \text { UCON oil }+80 \% \text { water } & 42 \pm 0.5 & 1.030 \pm 0.005 \\ 3 & 25 \% \text { UCON oil }+75 \% \text { water } & 97 \pm 6 & 1.040 \pm 0.005 \\ 4 & 30 \% \text { UCON oil }+70 \% \text { water } & 127 \pm 4 & 1.046 \pm 0.005 \\ 5 & 34 \% \text { UCON oil }+66 \% \text { water } & 202 \pm 6 & 1.051 \pm 0.005 \\ 6 & 37 \% \text { UCON oil }+63 \% \text { water } & 295 \pm 11 & 1.062 \pm 0.005\end{array}$

TABLE 2 . Fluid characteristics at $T \approx 20^{\circ} \mathrm{C}$. The viscosity $\mu$ was measured with a falling-ball viscometer and the fluid density $\rho_{f}$ with a volumetric flask of $20 \mathrm{ml}$.

with the same suspending fluid. The clouds with the largest radii were produced in the wider vessel of cross-section $20 \mathrm{~cm} \times 20 \mathrm{~cm}$. Since we had to track both slowand fast-evolving clouds, we used two different cameras. The shape evolution of the slow-evolving clouds was recorded by a digital video camera (Canon XM2) mounted on a vertical sliding rail with an acquisition rate of 25 frames per second and a resolution of $1300 \times 1030$ pixels. These clouds were illuminated by two neon tubes located on each side of the vessel and were shot in front of a black background. For the fast-evolving clouds, a high-speed camera (Photron Fastcam APX RS) with an acquisition rate up to 500 frames per second and a resolution of $1024 \times 1024$ pixels was required. These clouds were backlit using a $500 \mathrm{~W}$ spotlight through a diffusing paper.

Each frame was thresholded, and the cloud contour was fitted with an ellipse with ImageJ (a digital imaging software available at http://rsbweb.nih.gov/ij/). This process provided the position of the centre of mass as well as the horizontal $R$ and vertical $r$ radii (and thus the aspect ratio $\gamma=R / r$ ) of the cloud. The cloud velocity $V$ was measured from two successive images. The initial cloud velocity $V_{0}$ was measured over a set of successive images during which the cloud fell a distance of about five times its initial radius $R_{0}$. The initial number $N_{0}$ of particles was estimated from the known volume fraction $\phi$ and the measured radius $R_{0}$ by using $N_{0}=\phi\left(R_{0} / a\right)^{3}$. The radius $R_{0}$ and Stokes velocity $V_{0}^{\text {Stokes }}=N_{0} U_{0}\left(6 a / 5 R_{0}\right)$ (see $\S 4.2$ ) of the initially spherical cloud were used to normalise the data.

Four batches of spherical particles having different radii $a$ and densities $\rho_{p}$ were used in the experiments; their characteristics are given in table 1 . They were suspended in various mixtures of UCON oil and water having different viscosities $\mu$; their characteristics are given in table 2. Combining the different batches of particles and fluid mixtures enabled us to examine the low- to small-Reynolds-number regimes of evolution for a sedimenting cloud, as depicted in figure 1. The different sets of experiments could be approximately classified into three groups: the first group 
(light-grey symbols) for which $R e_{c} \lesssim 0.1$ and $\ell / R_{0} \gtrsim 10$ lay in the Stokes regime; the second group (dark-grey symbols) for which $R e_{c} \gtrsim 0.1$ and $\ell / R_{0} \gtrsim 10$ lay in the regime in which macro-scale inertia was dominant; and the third group (black symbols) for which $R e_{c} \gtrsim 0.1$ and $\ell / R_{0} \lesssim 10$ lay in the regime in which micro-scale inertia was dominant, i.e. in which the Oseen interactions were dominant. Because of the fast settling of the particles during the suspension preparation, only clouds having $\phi \approx 50 \%$ (circles in figure 1) were produced for $R e_{p} \gtrsim 10^{-3}$, while clouds having smaller $\phi \approx 2-10 \%$ (triangles in figure 1 ) were obtained for smaller $R e_{p}$. Note that in all the experiments the Stokes number $S t=(2 / 9)\left(\rho_{p} / \rho_{f}\right) R e_{p} \ll 1$, and thus the particles closely followed the fluid streamlines.

\section{Oseenlet simulations}

Following Subramanian \& Koch (2008), we consider that the particles within the cloud are driven by far-field steady Oseen interactions; i.e. the motion of each particle is due to the sum of the steady Oseen disturbances produced by all other particles. Despite the fact that the assumption of steady Oseen interactions for the particles within the cloud (and consequently the assumption of linearity) is not fully justified, we are following this approach because it leads to simple kinematic simulations, which provide some insight into the dynamics of sedimenting clouds.

Within the steady Oseen approximation, each particle of radius $a$ sedimenting with velocity $U_{0}$ creates a disturbance at a distance $r$ from its centre given by the Oseen stream function (see e.g. Lamb 1932, §342)

$$
\Psi=U_{0} a^{2}\left[-\frac{1}{4} \frac{a}{r} \sin ^{2} \theta+\frac{3}{2}(1-\cos \theta) \frac{1-\exp \left[-(1+\cos \theta) \frac{r R e_{p}}{2 a}\right]}{R e_{p}}\right]
$$

in a spherical coordinate system with the polar axis coincident with the direction of translation. In this leading-order Oseen approximation, the velocity scale is $U_{0}$, and the drag force is still the Stokes drag $-6 \pi \mu a U_{0}$, but there are two length scales, namely the sphere radius $a$ and the inertial length $\ell=a / R e_{p}=v / U_{0}$. The first term on the right-hand side of (3.1) is the finite-size portion, which happens to be identical to that of the Stokes solution. The second term is the flow induced by a point force, also called the Oseenlet. It reduces to the Stokeslet, $\Psi \rightarrow U_{0} a^{2} \sin ^{2} \theta(3 r / 4 a)$, when $r / \ell \rightarrow 0$. For $r \gg \ell$, the fore-aft symmetry of the Stokes flow structure is lost. The flow in the far field is radial except in a narrow wake (bounded by a paraboloidal surface) located directly behind the sphere, where the $(-1 / r)$ inflow compensates for the radial $\left(1 / r^{2}\right)$ outward flow. It is more convenient for the following analysis to express the Oseenlet in Cartesian coordinates,

$$
u_{i}=\frac{F}{8 \pi \mu}\left\{\frac{x_{i}}{r^{2}}\left[\frac{2 \ell}{r}(1-E)-E\right]+\frac{E}{r} \delta_{i 3}\right\},
$$

where $F\left(\equiv 6 \pi \mu a U_{0}\right)$ is the magnitude of the buoyancy force that acts along the direction $i=3$ and $E=\exp \left(-\left(1+x_{3} / r\right) r / 2 \ell\right)$.

Since the steady Oseen equations are still linear (but no longer reversible), the velocity $\dot{r}_{i}^{\alpha}$ of a point particle $\alpha=1, \ldots, N_{0}$, located at $r_{i}^{\alpha}$ within the cloud, is equal to the sum of its terminal velocity $U_{0} \delta_{i 3}$, when in isolation, and the fluid velocity 
disturbances, i.e. the Oseenlets, generated by all the other point particles $\beta$,

$$
\frac{\mathrm{d} r_{i}^{\alpha}}{\mathrm{d} t}=U_{0} \delta_{i 3}+\frac{F}{8 \pi \mu} \sum_{\alpha \neq \beta}\left\{\frac{r_{i}}{r^{2}}\left[\frac{2 \ell}{r}(1-E)-E\right]+\frac{E}{r} \delta_{i 3}\right\},
$$

where $r_{i}=r_{i}^{\alpha}-r_{i}^{\beta}$ and $r$ is the scalar length between the centres of particles $\alpha$ and $\beta$. We can then proceed as in previous Stokeslet simulations for falling clouds (EkielJeżewska, Metzger \& Guazzelli 2006; Metzger et al. 2007) by choosing the frame of reference moving with the terminal settling velocity of an isolated particle and deciding to make all the values dimensionless by scaling the length and the velocity with the radius $R_{0}$ and the Stokes velocity $V_{0}^{\text {Stokes }}=N_{0} F / 5 \pi \mu R_{0}$ of the initially spherical cloud of point particles, respectively, in the same way as in the experiments (note that this Stokes velocity corresponds to that of a dilute cloud experiencing a drag force $-5 \pi \mu R_{0} V_{0}^{\text {Stokes }}$ and is set as the reference velocity in both the experiments and the simulations). The set of equations (3.3) becomes

$$
\frac{\mathrm{d} r_{i}^{* \alpha}}{\mathrm{d} t^{*}}=\frac{5}{8 N_{0}} \sum_{\alpha \neq \beta}\left\{\frac{r_{i}^{*}}{r^{* 2}}\left[\frac{2 \ell^{*}}{r^{*}}(1-E)-E\right]+\frac{E}{r^{*}} \delta_{i 3}\right\},
$$

where the superscript * denotes dimensionless quantities. In this reference frame and with this normalisation, the set of equations (3.4) possesses two parameters: the number of particles $N_{0}$ and the normalised inertial length $\ell^{*}=\ell / R_{0}$. The corresponding Stokeslet set of equations has only a single parameter $N_{0}$ (Metzger et al. 2007). It is worth mentioning that the simulations based on (3.4) allow continuous progress from a zero-inertia (Stokes) regime to a micro-scale-inertia regime by simply decreasing the inertial length.

In the numerical simulations, the $N_{0}$ particles were initially randomly distributed inside a sphere of dimensionless radius $R_{0}^{*}=1$. The positions were then integrated using a variable-order Adams-Bashforth-Moulton solver (ode113 in Matlab). These simulations were performed with inertial length $0.5 \leqslant \ell^{*} \leqslant 1000$ and with initial spherical clouds comprising $500 \leqslant N_{0} \leqslant 7500$ particles, which were tracked over a typical time interval $0 \leqslant t^{*} \leqslant 1200$ (in particular for the simulations having the largest $\ell^{*}$, which had to run for a longer time to reach breakup). Because large variations exist from one run to the next for the same couple of parameters $N_{0}$ and $\ell^{*}$, typically five runs for different realisations of the initial particle positions were performed and were found to be sufficient to provide a meaningful average and standard deviation.

For each run, the position of the centre of mass of the cloud was identified, and measuring its displacement between two successive times $t^{*}$ and $t^{*}+1$ provided the cloud velocity $V^{*}\left(t^{*}\right)$. The particles were considered to belong to the cloud at time $t^{*}$ if their vertical position from the centre of mass was $\leqslant R_{0}$. This yielded the number of particles staying inside the cloud, $N\left(t^{*}\right)$. For a direct comparison between simulations and experiments, we chose to follow Metzger et al. (2007) for the definition of the horizontal and vertical radii. The vertical radius $r\left(t^{*}\right)$ was defined as the distance from the front leading particle to the centre of mass of the cloud. The horizontal radius $R\left(t^{*}\right)$ was defined as the average of the maximum distance from the centre of mass over four quadrants in the horizontal plane. This whole procedure generated an ensemble of data over which to average the macroscopic quantities of the cloud such as the cloud velocity $V^{*}$, the number $N$ of particles staying in the cloud and its horizontal-to-vertical aspect ratio $\gamma$, as function of time $t^{*}$. The dispersion of the data among runs was simply provided by the standard deviation. 
(a)
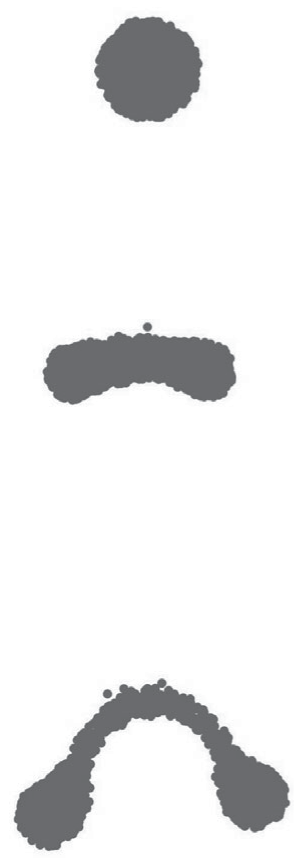

(b)
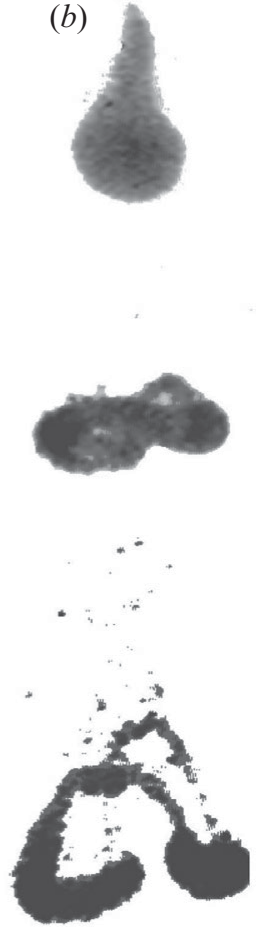

FiguRE 2. Typical evolution of a cloud in the macro-scale-inertia regime: $(a)$ the simulations of Bosse et al. $(2005 a, b)$ with $R e_{c}=1, N_{0}=100096$ and $\ell^{*}=2600$ and $(b)$ the experiment with $R e_{c}=0.7, N_{0}=16000$ and $\ell^{*}=179\left(R e_{p}=8 \times 10^{-5}, R_{0} / a=66, \phi=6 \%\right.$ and $\left.S t=2 \times 10^{-5}\right)$. The complete dynamics for the experimental case is shown in supplementary movie 1 (available at journals.cambridge.org/flm). The movie of the numerical simulation can be seen in Bosse et al. (2005a). The upward tail of the experimental cloud at initial time is due to the injection process.

\section{Results and comparison}

\subsection{General evolution}

The typical evolution of a cloud in the regime of macro-scale inertia is displayed in figure 2. The cloud is seen to flatten and to transition into a toroidal shape. The formed torus further expands and eventually breaks up into two droplets which may destabilise again in a repeating cascade if the cloud contains a large enough number of particles. No significant leakage of particles from the rear of the cloud is observed. Similar qualitative evolution is found between the simulation of Bosse et al. $(2005 a, b)$ and the experiments within this regime.

The typical evolution in the regime of micro-scale inertia when the inertial length is of the order of the size of the cloud, i.e. $\ell^{*} \approx 1$, is shown in figure 3 . The cloud is also seen to flatten and to become a torus which further widens and eventually shatters into two or three droplets. Again, there is no particle leakage. Conversely, when the inertial length is increased (see figure 4 with $\ell^{*} \approx 20$ ), some leakage is observed, and the sequence of events (transition towards a torus and breakup) resembles more that observed for a Stokes cloud. Good qualitative agreement is seen between the experiments and the Oseenlet simulations. Overall, the evolution rate increases with decreasing inertial length. 
(a)
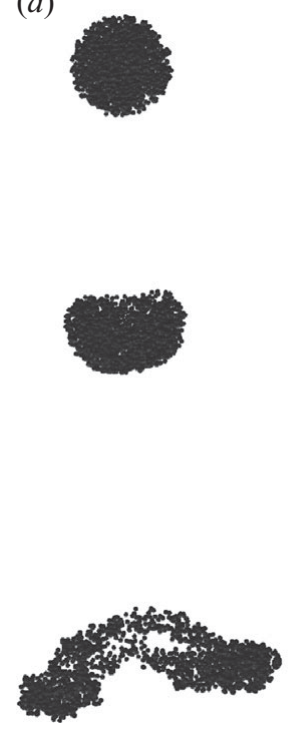

(b)
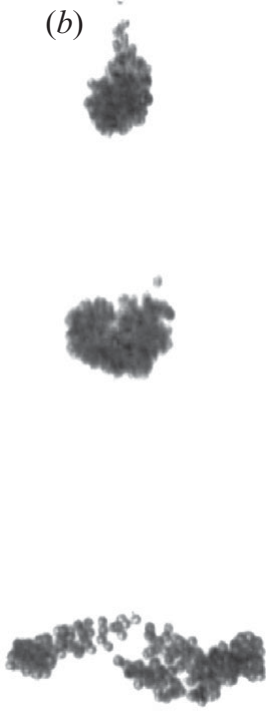

FIGURE 3. Typical evolution of a cloud in the micro-scale-inertia regime $\ell^{*} \approx 1:(a)$ the Oseenlet simulations with $N_{0}=2000$ and $\ell^{*}=1$ and $(b)$ the experiment with $R e_{c}=15, N_{0}=600$ and $\ell^{*}=0.65\left(R e_{p}=0.14, R_{0} / a=11, \phi \approx 50 \%\right.$ and $\left.S t=0.077\right)$. The complete dynamics for the experimental case is shown in supplementary movie 2 and for the Oseenlet simulation in supplementary movie 4 . The small upward tail of the experimental cloud at initial time is due to the injection process.

(a)
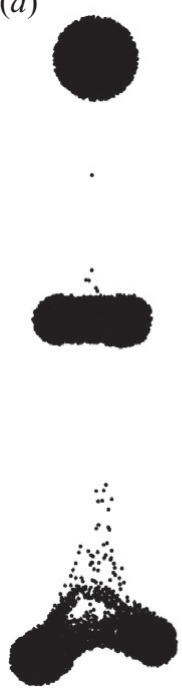

(b)
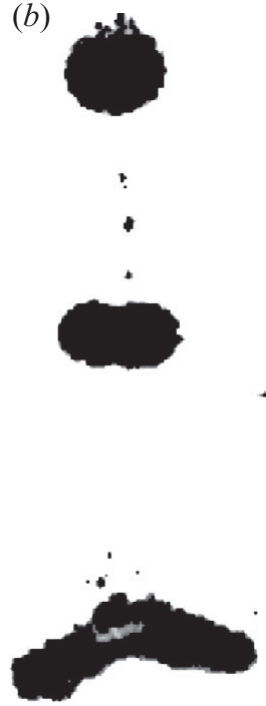

FIGURE 4. Typical evolution of a cloud in the micro-scale-inertia regime for $\ell^{*} \approx 20$ : $(a)$ the Oseenlet simulations with $N_{0}=7500$ and $\ell^{*}=20$ and $(b)$ the experiment with $R e_{c}=3.5$, $N_{0}=7000$ and $\ell^{*}=21\left(R e_{p}=2 \times 10^{-3}, R_{0} / a=24, \phi \approx 50 \%\right.$ and $\left.S t=5 \times 10^{-4}\right)$. The complete dynamics for the experimental case is shown in supplementary movie 3 and for the Oseenlet simulation in supplementary movie 5. 


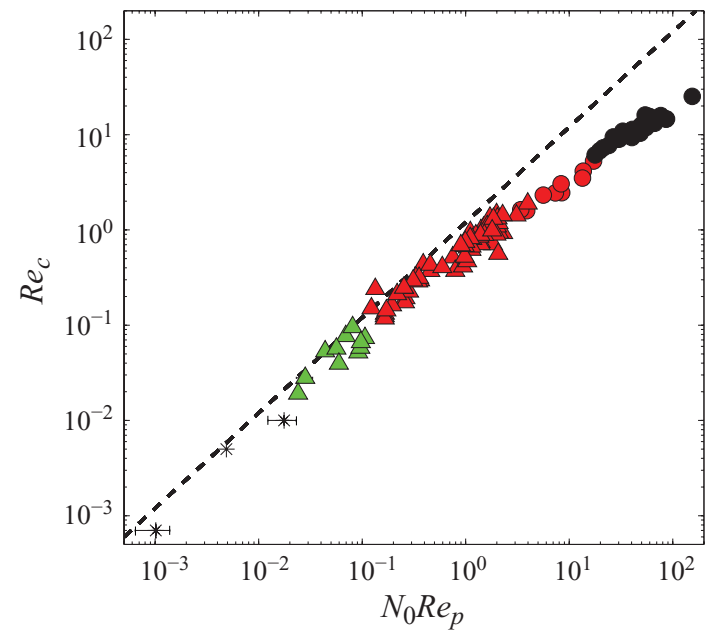

FIGURE 5. The Reynolds number of the experimental cloud, $R e_{c}$, versus the product of the initial number of particles by the particle Reynolds number, $N_{0} R e_{p}$. The dashed line denotes $R e_{c}=6 N_{0} R e_{p} / 5$. The symbols are the same as in figure 1 . The error bars are of the same size as or smaller than the symbols.

In all the above evolutions, the particles present a toroidal circulation inside the cloud, which can be clearly seen in the supplementary movies.

\subsection{Cloud settling velocity and aspect ratio}

We start by examining the initial velocity $V_{0}$ of the cloud in both the experiments and the Oseenlet simulations. In the Stokes regime, $V_{0}^{\text {Stokes }} \sim N_{0} U_{0} a / R_{0}$. In the experiments, the deviation of $V_{0}$ from this Stokes scaling with increasing inertia is shown in figure 5 by plotting the cloud Reynolds number $\operatorname{Re}_{c}=V_{0} R_{0} \rho_{f} / \mu$ versus the product of the initial number $N_{0}$ of particles by the particle Reynolds number $R e_{p}=U_{0} a \rho_{f} / \mu$. For $R e_{c} \lesssim 1$, the experimental data follow approximately the Stokes scaling $\approx N_{0} U_{0}\left(6 a / 5 R_{0}\right)$. For $R e_{c} \gtrsim 1, V_{0}$ becomes increasingly lower than $V_{0}^{\text {Stokes }}=N_{0} U_{0}\left(6 a / 5 R_{0}\right)$ with increasing $R e_{c}$. In the Oseenlet simulations, the deviation of $V_{0}$ from $V_{0}^{\text {Stokes }}$ for an initially spherical cloud of point particles is shown in figure 6 as a function of the normalised inertial length $\ell^{*}$. For $\ell^{*} \gtrsim 50, V_{0} \approx V_{0}^{\text {Stokes }}$, while for $\ell^{*} \lesssim 50, V_{0}$ becomes increasingly lower than $V_{0}^{\text {Stokes }}$ with decreasing $\ell^{*}$. Note that normalising $V_{0}$ by $V_{0}^{\text {Stokes }}$ makes the data independent of $N_{0}$. It should be mentioned that, while $V_{0}$ is a quantity directly measured in the experiments and simulations, $U_{0}$ is calculated from the particle properties.

As time increases, the cloud flattens and evolves towards a torus which expands, and consequently its aspect ratio increases while its velocity decreases. This general trend is observed in regimes of both macro- and micro-scale inertia as seen in figures 7 and 8.

Comparison between experimental data in the regime of macro-scale inertia (symbols) and the numerical simulations of Bosse et al. $(2005 a, b)$ is shown in figure 7 . The numerical data have been inferred from the movie shown in Bosse et al. (2005a) by an analysis similar to that described in $\S 2$ for the experimental movies. While the rate of decrease of $V^{*}$ is similar in the experiments and the simulations, the rate of growth of $\gamma$ is stronger in the experiments than in the simulations. Note also the difference in normalised initial velocity between the experiments and the simulations, indicating differences in deviation from the Stokes scaling. 


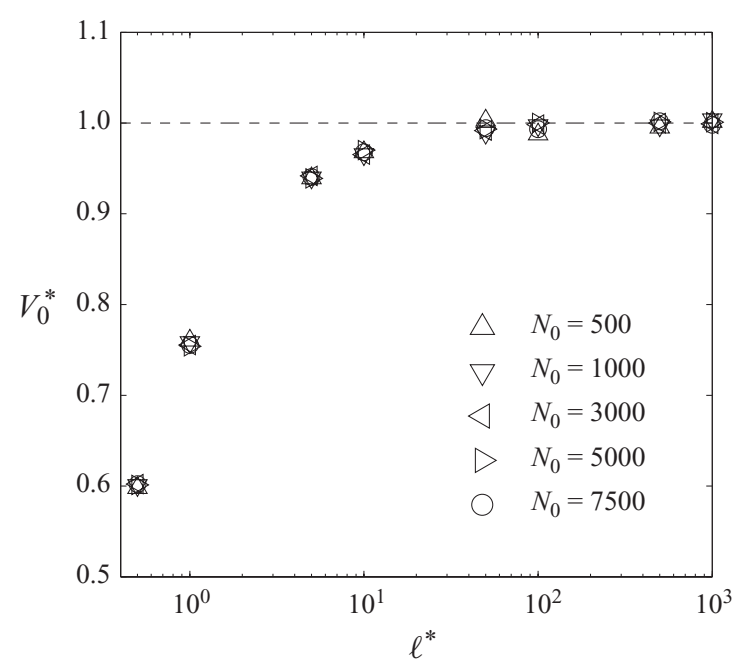

FIGURE 6. Numerical initial velocity $V_{0}$ normalised by the Stokes velocity $V_{0}^{\text {Stokes }}=$ $N_{0} U_{0}\left(6 a / 5 R_{0}\right)$ versus normalised inertial length $\ell^{*}$. The error bars are smaller than the size of the symbols.
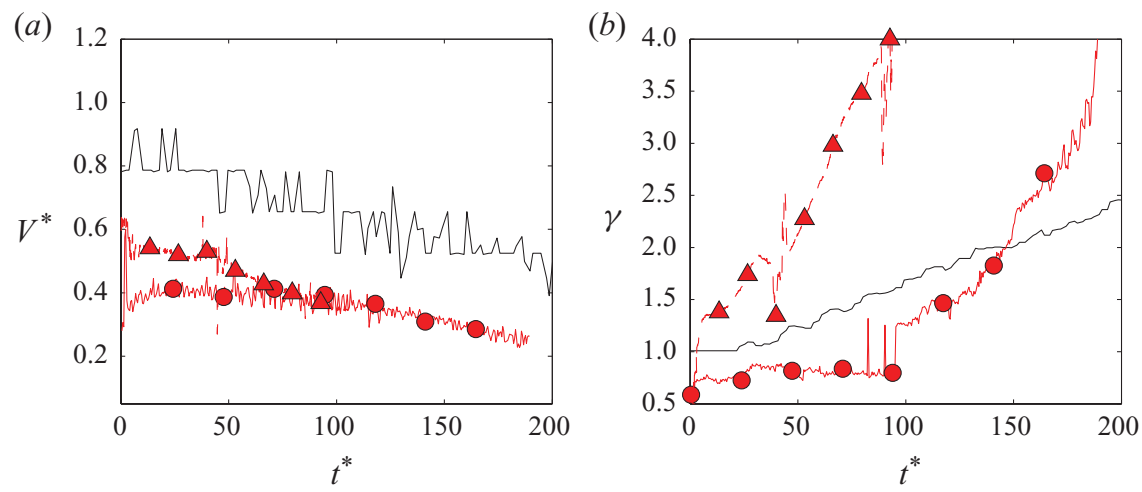

Figure 7. (a) Cloud velocity $V^{*}$ and $(b)$ aspect ratio $\gamma$ versus time $t^{*}$ for the following: simulation of Bosse et al. $(2005 a, b)$ at $R e_{c}=1$, denoted by the solid line; experiment with $R e_{c}=1.08, N_{0}=19000$ and $\ell^{*}=185\left(R e_{p}=8 \times 10^{-5}, R_{0} / a=64, \phi \approx 8 \%\right.$, and $\left.S t=2 \times 10^{-5}\right)$, denoted by dark-grey $\triangle$ with the dotted line; experiment with $R e_{c}=1.6, N_{0}=1780$ and $\ell^{*}=34$ $\left(R e_{p}=0.0019, R_{0} / a=15, \phi \approx 50 \%\right.$ and $\left.S t=5 \times 10^{-4}\right)$, denoted by dark-grey $\circ$ with the solid line.

Comparison between the experimental data and the Oseenlet simulations is shown in figure 8. For $\ell^{*} \gtrsim 1000$, the evolution of $V^{*}$ and $\gamma$ is similar to that obtained in the Stokes regimes. In particular, the Oseenlet simulations (in figures $8 e-f$ ) give results similar to those of the Stokeslet simulations (in figures $8 g-h$ ). The rate of decrease of $V^{*}$ and to a lesser extent the rate of increase of $\gamma$ are stronger for smaller $N_{0}$. The agreement between the experiments and the simulations is good as far as the evolution of the rate of decrease of $V^{*}$ is concerned (see figure $8 e$ ). A stronger growth of $\gamma$ is observed in the experiments as previously reported by Metzger et al. (2007) (see figure $8 f$ ). As a general trend, when $\ell^{*}$ is reduced, the rate of decrease of $V^{*}$ and the rate of growth of $\gamma$ increases, and both tend to become independent of $N_{0}$ (see figure $8 a-d$ ). Good agreement between the experiments and the simulations is obtained for the rate of decrease of $V^{*}$ and the rate of growth of $\gamma$ at $\ell^{*}=20$. Some 

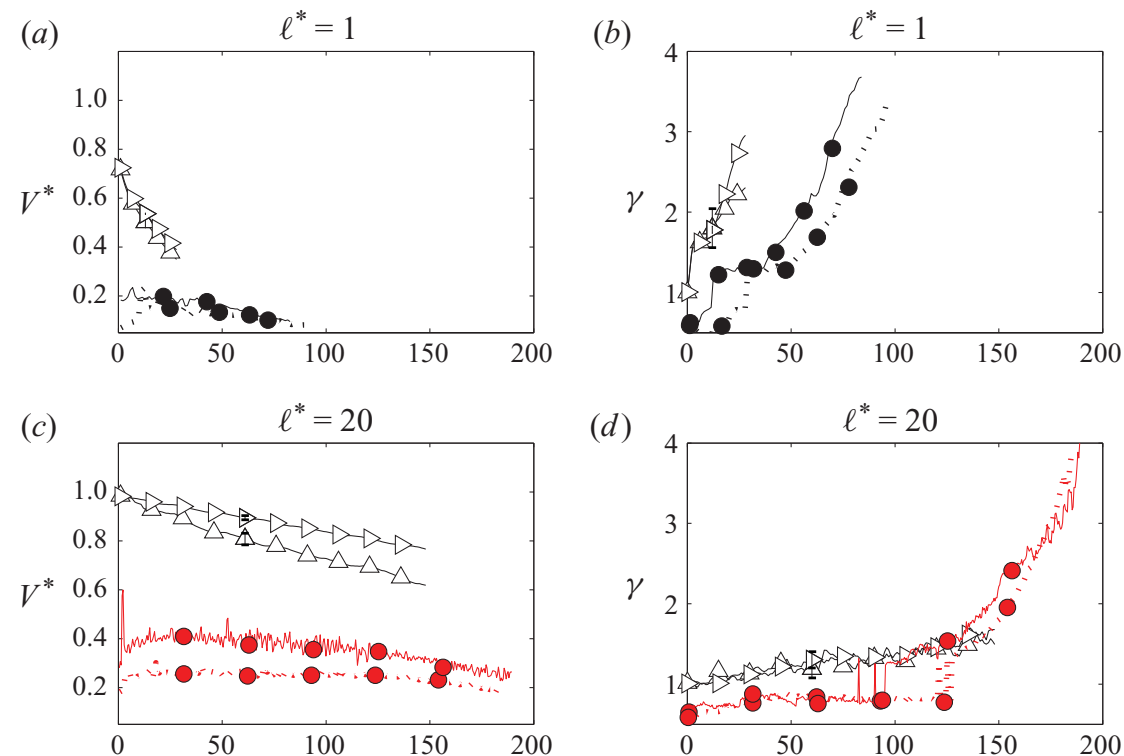

(d)
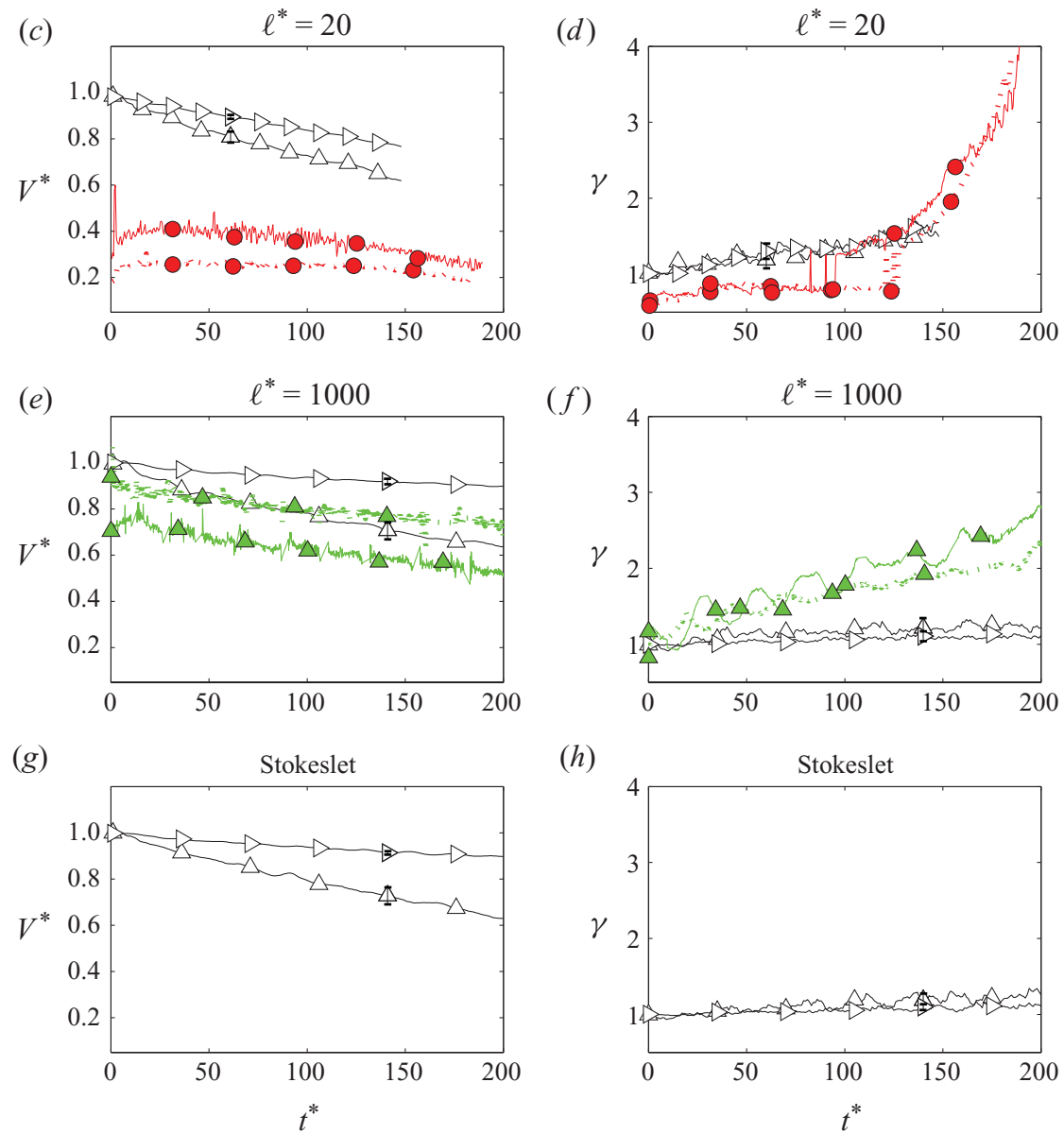

Figure 8. $(a, c, e, g)$ Cloud velocity $V^{*}$ and $(b, d, f, h)$ aspect ratio $\gamma$ versus time $t^{*}$ for different $\ell^{*}$. Simulations in all graphs: $\triangle, N_{0}=500 ; \triangleright, N_{0}=5000$. Experiments in $(a)$ and $(b)$ : black $\bigcirc$ with solid line, $R e_{c}=11.4, N_{0}=340$ and $\ell^{*}=0.79\left(R e_{p}=0.14, R_{0} / a=9, \phi \approx 50 \%\right.$ and $S t=0.077)$; black $\bigcirc$ with dotted line, $R e_{c}=14.6, N_{0}=602$ and $\ell^{*}=0.65\left(R e_{p}=0.14, R_{0} / a=11\right.$, $\phi \approx 50 \%$ and $S t=0.077$ ). Experiments in $(c)$ and $(d)$ : dark-grey $\bigcirc$ with solid line, $R e_{c}=1.6$, $N_{0}=1768$ and $\ell^{*}=34\left(R e_{p}=0.0019, R_{0} / a=15, \phi \approx 50 \%\right.$ and $\left.S t=5 \times 10^{-4}\right)$; dark-grey 0 with dotted line, $R e_{c}=5.3, N_{0}=8935$ and $\ell^{*}=20\left(R e_{p}=0.0019, R_{0} / a=26, \phi \approx 50 \%\right.$ and $\left.S t=5 \times 10^{-4}\right)$. Experiments in $(e)$ and $(f)$ : light-grey $\triangle$ with solid line, $R e_{c}=0.066, N_{0}=700$ and $\ell^{*}=340\left(R e_{p}=1.4 \times 10^{-4}, R_{0} / a=21, \phi \approx 8 \%\right.$ and $\left.S t=7 \times 10^{-5}\right)$; light-grey $\triangle$ with dotted line, $R e_{c}=0.78, N_{0}=4040$ and $\ell^{*}=1575\left(R e_{p}=1.7 \times 10^{-5}, R_{0} / a=37, \phi \approx 8 \%\right.$ and $\left.S t=9 \times 10^{-6}\right)$. Stokeslet simulations in $(g)$ and $(h)$. The error bars are of the same size as or smaller than the symbols. 


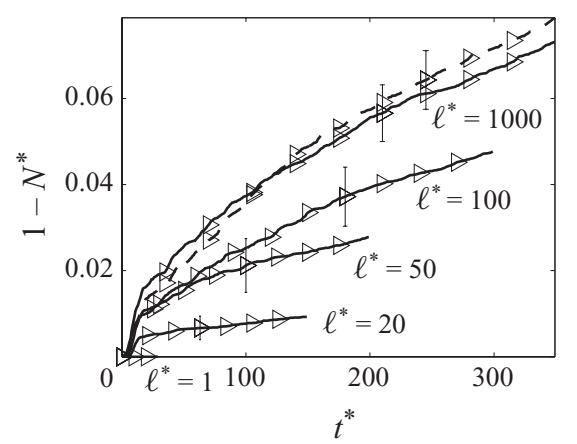

FIGURE 9. Percentage $1-N^{*}$ of particles that have leaked away from the cloud as a function of time $t^{*}$ for $N_{0}=5000(\triangleright)$. The dispersion of the numerical data (one standard deviation) is indicated for a few instants. The solid lines denote the Oseenlet simulations at different $\ell^{*}$ indicated in the graph. The dashed line denotes the Stokeslet simulation.

divergence is obtained at $\ell^{*}=1$, in particular for the rate of decrease of $V^{*}$. Note again that the deviation of the initial velocity $V_{0}$ from the Stokes scaling is stronger in the experiments.

\subsection{Particle leakage}

While particle leakage is an important feature at zero Reynolds number (Nitsche \& Batchelor 1997; Metzger et al. 2007), no leakage is observed in the regime of macroscale inertia (see figure 2, supplementary movie 1 and the movie shown in Bosse et al. $2005 a$ ) and that of micro-scale inertia for $\ell^{*} \sim 1$ (see figure 3 and supplementary movies 2 and 4). When $\ell^{*}$ is increased (see figure 4 and supplementary movies 3 and $5)$, the rate of leakage increases, and the cloud evolution tends to be similar to that found at zero Reynolds number.

A precise evaluation of the rate of leakage is not readily feasible in the experiments. The fast settling of the particles due to inertia causes difficulty in the injection process. Consequently, the initial clouds are not perfectly spherical, and a strong leakage is seen at early times. This leads to problems in obtaining any accurate measurement. We have thus chosen to rely upon the Oseenlet simulations to study the variation of the rate of leakage with inertia.

Figure 9 shows the percentage $1-N^{*}$ of particles that have leaked away from the cloud as a function of time $t^{*}$ for a fixed initial number of particles, $N_{0}=$ 5000 , but for varying inertial lengths, $1 \leqslant \ell^{*} \leqslant 1000$. As $\ell^{*}$ is increased, the particle leakage increases and tends to that predicted by the Stokeslet simulation for $\ell^{*} \gtrsim 1000$. This shows that increasing the inertial length in the Oseenlet simulations provides a continuous transition from a micro-scale-inertia-dominated regime to a Stokesdominated regime.

As found for Stokes clouds (Metzger et al. 2007), two different regimes having two different rates of leakage are observed. For $t^{*} \lesssim 10$ (when the cloud shape is still approximately spherical), the rate of leakage is large, while for $t^{*} \gtrsim 10$ (when the cloud has evolved towards a toroidal shape), the rate of leakage is lower. Note that for $\ell^{*} \lesssim 5$, these two regimes are not seen because there is nearly no leakage.

One can estimate the averaged rate of leakage $\left\langle-\mathrm{d} N^{*} / \mathrm{d} t^{*}\right\rangle$ for $t^{*} \gtrsim 10$ by defining $\left\langle-\mathrm{d} N^{*} / \mathrm{d} t^{*}\right\rangle=\left(N(10)-N\left(t_{f}^{*}\right)\right) /\left(t_{f}^{*}-10\right)$, where $t_{f}^{*}$ is taken as large as possible. In figure $10(a),\left\langle-\mathrm{d} N^{*} / \mathrm{d} t^{*}\right\rangle$ is plotted versus $N_{0}$ for several $\ell^{*}$ in the log-log scale. Again, for large $\ell^{*}$ (typically $\ell^{*} \gtrsim 50$ ), the dependence of the rate of leakage on $N_{0}$ 
(a)

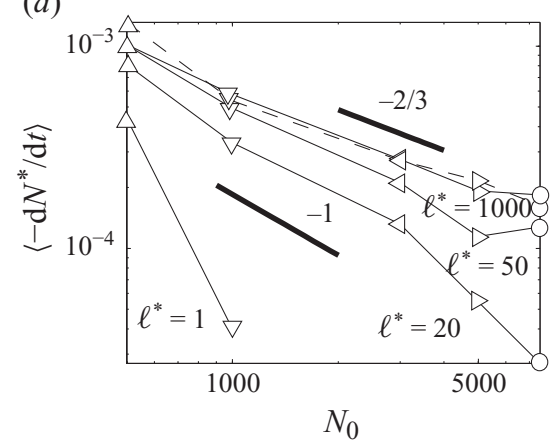

(b)

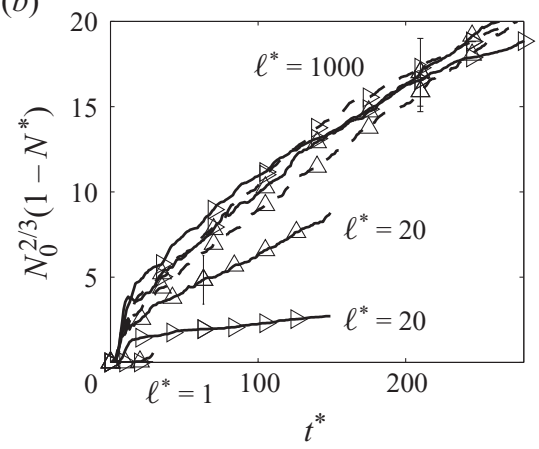

Figure 10. (a) Rate of leakage $\left\langle-\mathrm{d} N^{*} / \mathrm{d} t^{*}\right\rangle$ estimated for $t^{*}>10$ versus $N_{0}$ obtained from the Oseenlet simulations (solid lines) for four different inertial lengths indicated in the graph $\left(\ell^{*}=1,20,50,1000\right)$ and from the Stokeslet simulations (dashed line). The lines are a guide to the eye. (b) $N_{0}^{2 / 3}\left(1-N^{*}\right)$ as a function of $t^{*}$. The dispersion of the numerical data (one standard deviation) is indicated for a few instants. The solid lines denote the Oseenlet simulations at different $\ell^{*}$ indicated in the graph. The dashed line denotes the Stokeslet simulation. The symbols indicate different $N_{0}$, as in figure 6 .

is similar to that predicted by the Stokeslet simulations of Metzger et al. (2007), and one finds a scaling $\propto N_{0}^{-2 / 3}$. For lower $\ell^{*}$, the dependence on $N_{0}$ differs. As an attempt at obtaining a better collapse of the data, we have plotted in figure $10(b) N_{0}^{2 / 3}\left(1-N^{*}\right)$ as a function of $t^{*}$ for $N_{0}=500$ and 5000 and for different $\ell^{*}$. For $\ell^{*} \gtrsim 50$, the dependence of $\left(1-N^{*}\right)$ on $N_{0}$ is correctly approximated by the scaling $\propto N_{0}^{-2 / 3}$, and the data obtained for different $N_{0}$ gather on the same curve, whereas for lower $\ell^{*}$, the dependence on $N_{0}$ differs, and the data obtained for different $N_{0}$ are clearly separated.

\subsection{Breakup}

A physical picture of the mechanisms affecting the particle leakage, the evolution towards a torus and the breakup can be obtained from a visualisation of the flow field inside and outside the cloud.

When macro-scale inertia is dominant, Bosse et al. (2005b) have shown that the initially spherical cloud flattens into an oblate shape featuring a growing dimple at its rear, which eventually leads to the later evolution towards a torus. The different flow fields in figure 8 of their paper show the breakup transition from a closed toroidal vortex to an open ring vortex in which streamlines start to pass through the cloud centre hole, anticipating the torus disintegration.

Similar features are observed in the regime in which micro-scale inertia is dominant. Figure 11 shows the successive flow fields computed in a vertical plane passing through the vertical axis of symmetry in the instantaneous reference frame of the cloud for $\ell^{*}=1$ and 20 . This vertical plane is meshed, and the local velocities are obtained by summing the velocity disturbances (Oseenlets) of all the particles.

The source-wake character of the Oseen flow field at the micro-scale is a fundamental ingredient for the understanding of the evolution of the cloud, as it impacts on the flow field at the macro-scale. In particular, at early times, pure fluid is pulled inside the cloud from behind in a region close to the vertical axis, which leads to the further evolution towards a torus (see figure $11 a, c$ at $t^{*}=0$ and 17 for $\ell^{*}=1$ ). This mechanism for torus formation differs fundamentally from that observed in the Stokes regime. While in the Stokes regime, torus formation is due to particle depletion near the vertical axis caused by particle leakage (Metzger et al. 2007), here 


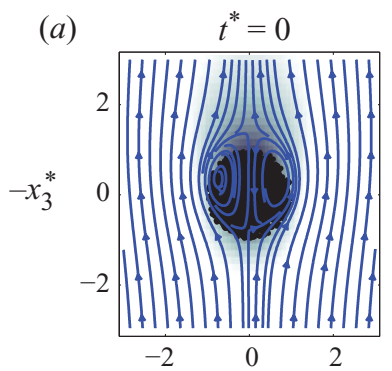

(b)
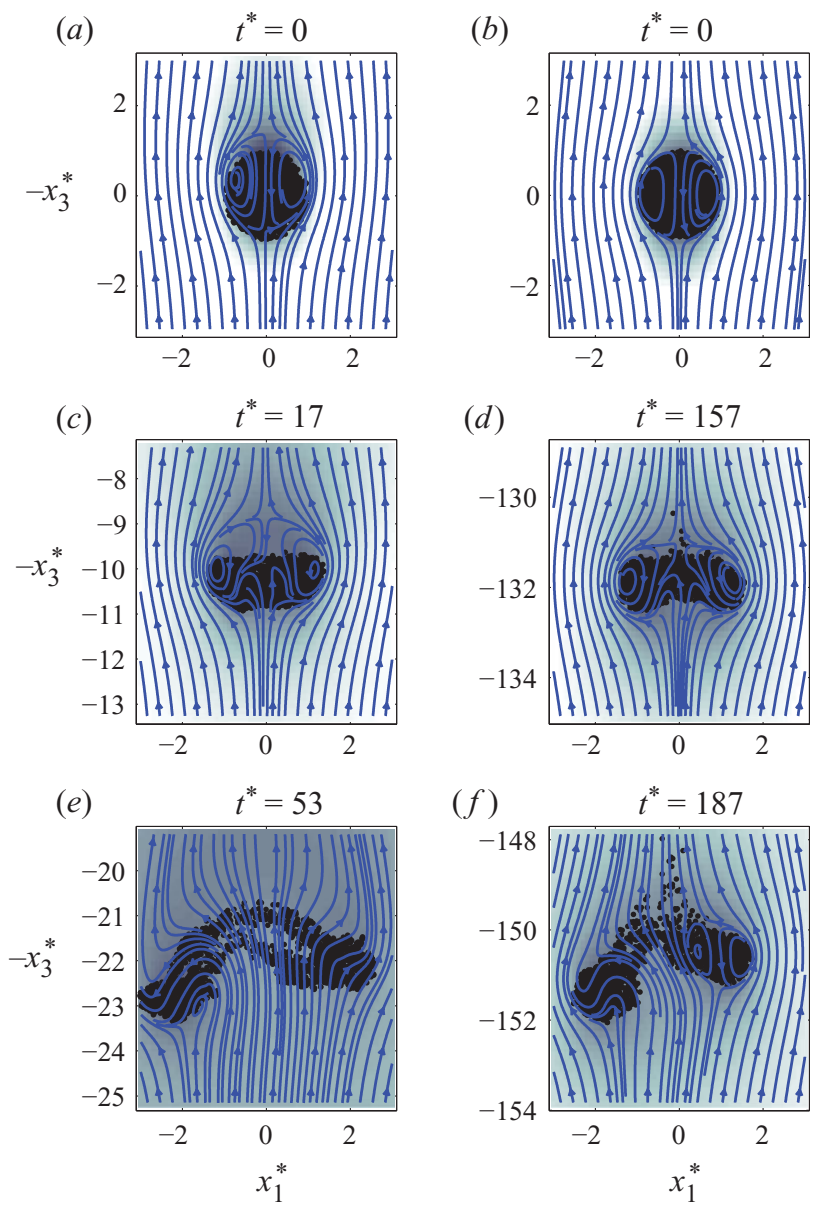

FIGURE 11. Flow field computed at successive times in the vertical plane through the vertical axis of symmetry in the cloud reference frame: $(a, c, e) \ell^{*}=1$ and $N_{0}=2000 ;(b, d, f) \ell^{*}=20$ and $N_{0}=2000$. High (low) velocity is indicated in white (dark). The complete dynamics is shown in supplementary movies 4 and 5 .

in the inertial regimes it is due to fluid inflow at the rear of the cloud. When inertia is increased, particle leakage is indeed drastically reduced, as the particles cannot escape from the cloud owing to this inflow. This inflow can also be detected in the experiments for $\ell^{*} \sim 1$, as particles belonging to the initial tail are caught back inside the settling cloud (see supplementary movie 2 ).

After the formation of the torus, the cloud expands laterally. Again, the breakup mechanisms in the inertial and Stokes regimes are dissimilar. While in the Stokes regime, in order to break up the cloud needs to reach a critical aspect ratio to allow streamlines to pass through its centre hole, here in the inertial regime, streamlines can go inside the cloud without instantaneously inducing breakup. As seen in figure 11(c) at $t^{*}=17$ for $\ell^{*}=1$, the front-incoming streamlines can enter the cloud, but the rearincoming streamlines are still pulling fluid inside, which prevents the front inflow from going all the way through the cloud. The same behaviour but with lesser strength is seen in figure $11(d)$ at $t^{*}=157$ for $\ell^{*}=20$. Consequently, the front streamlines are turning sideways. This increases the cloud's lateral expansion until a greater aspect 


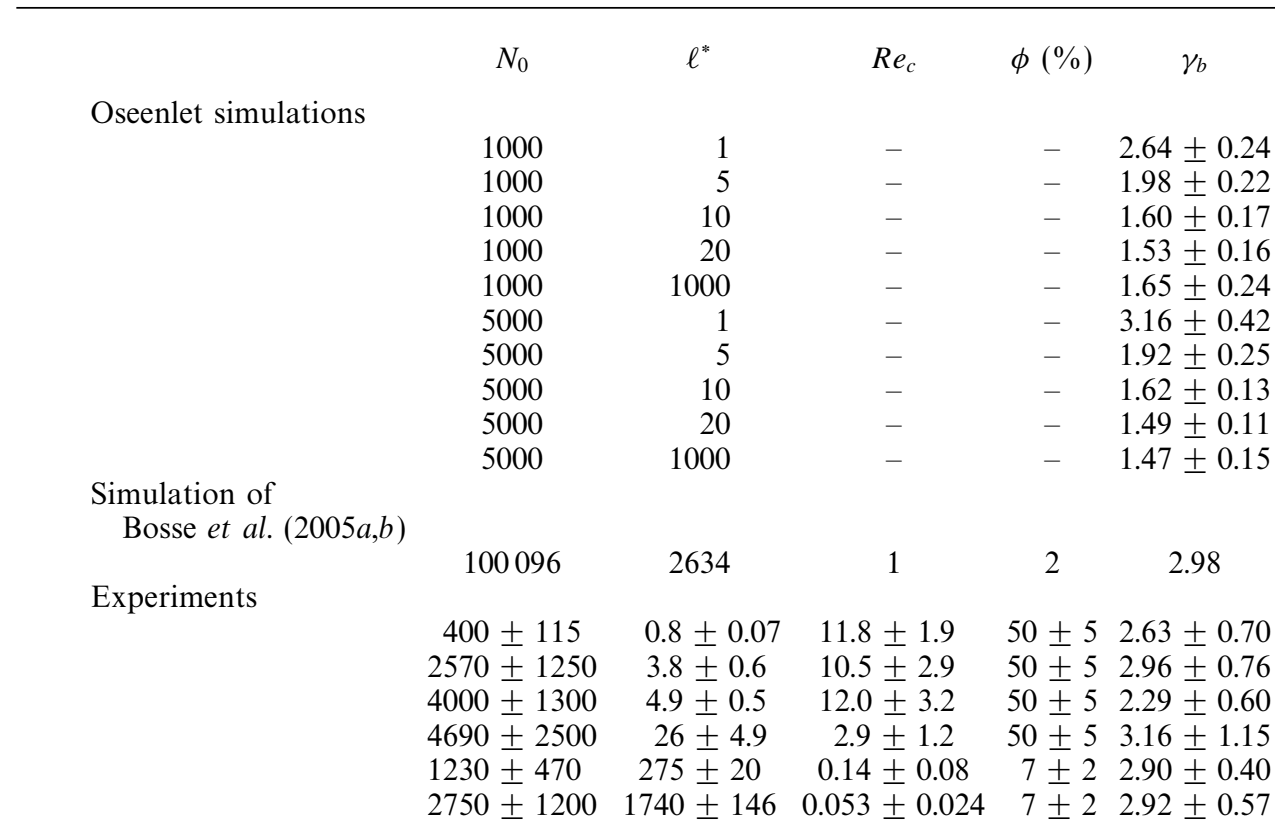

TABLE 3. The critical aspect ratio $\gamma_{b}$ at breakup for the Oseenlet simulations, the simulations of Bosse et al. $(2005 a, b)$ and the experiments. For the Oseenlet simulations, the uncertainty represents the dispersion (one standard deviation) over five runs. For the simulations of Bosse et al. (2005a,b), uncertainties are unknown, since only one simulation is available. For experimental data, uncertainties represent the dispersion (one standard deviation) obtained over different runs. For the Oseenlet simulations, data for $R e_{c}$ and $\phi$ are missing because these quantities require knowledge of the particle radius, which is missing in the point-particle simulations.

ratio, for which the front streamlines can finally poke through the cloud and induce breakup, is reached.

The values for the critical aspect ratio $\gamma_{b}$ at breakup are reported in table 3 for the Oseenlet simulations, for the available simulation of Bosse et al. $(2005 a, b)$ and for six different sets of experiments corresponding to the regimes identified in figure 1. Data coming from the Oseenlet simulations confirm the tendency to break up at larger $\gamma_{b}$ for smaller $\ell^{*}$ (note that data for $\operatorname{Re}_{c}$ and $\phi$ are not reported for the Oseenlet simulations because these quantities require knowledge of the particle radius, which is missing in the point-particle simulations). When macro-inertia is important, the simulation of Bosse et al. $(2005 a, b)$ indicates that breakup occurs for a large $\gamma_{b}=2.98$. Despite large scatters, the experimental data seem to present larger values than those of the Oseenlet simulations, except for $\ell^{*} \approx 0.8$ where the experimental $\gamma_{b}=2.63 \pm 0.70$ is in remarkable agreement with the numerical $\gamma_{b}=2.64 \pm 0.24$ (Oseenlet simulation for $N_{0}=1000$ and $\ell^{*}=1$ ).

Another important quantity which characterises the lifetime of the cloud is the breakup time $t_{b}$, i.e. the time for which the torus starts to bend to break up into secondary droplets. In the Stokes regime, the breakup time normalised by the Stokes time of the cloud, $t_{b}^{*}$, depends only on $N_{0}$. As explained in $\S 1$, when weak inertia is present (but still $S t \ll 1$ ), dimensional analysis indicates that there are two additional 
(a)

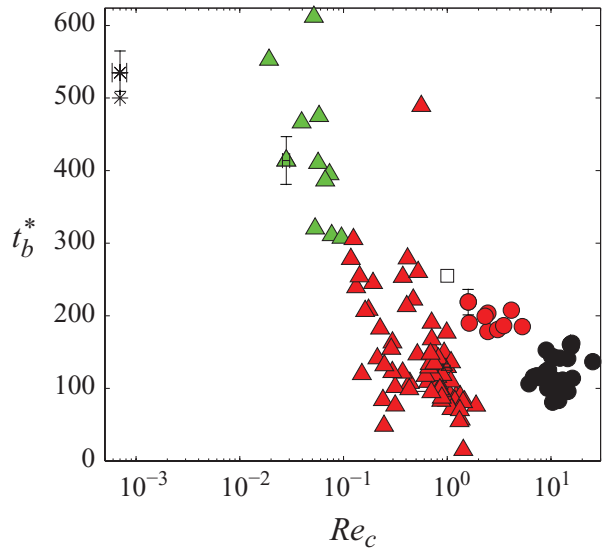

(b)

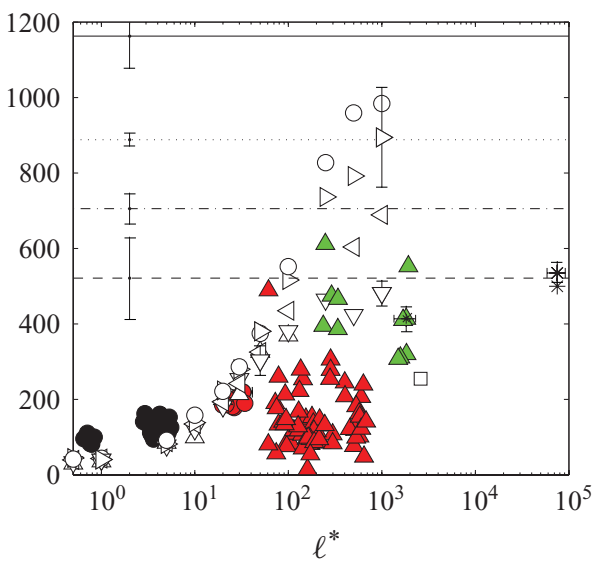

FIGURE 12. Normalised breakup time $t_{b}^{*}$ versus $(a)$ cloud Reynolds number $R e_{c}$ and $(b)$ normalised inertial length $\ell^{*}$. The symbols are the same as in figure 1 with the addition of those in figure 6 for the Oseenlet simulations with different $N_{0}$. The Stokeslet simulations with $N_{0}=1000$ are denoted as the dashed lines, those with $N_{0}=3000$ as the dash-dotted line, those with $N_{0}=5000$ as the dotted line and those with $N_{0}=7500$ as the solid line. The error bars are indicated for some data points.

dimensional numbers that we can choose conveniently to be the cloud Reynolds number $R e_{c}$ and the normalised inertial length $\ell^{*}$.

The normalised breakup time $t_{b}^{*}$ is plotted versus $R e_{c}$ in figure 12(a). At low $R e_{c}$, i.e. $R e_{c} \lesssim 0.1$, the data (light-grey triangle) gather on a single curve showing a decrease of $t_{b}^{*}$ with increasing $R e_{c}$. For $R e_{c} \gtrsim 0.1$, this curve divides into two branches. The lower branch contains the data located in the regime of macro-scale inertia (darkgrey triangles), while the upper branch comprises those belonging to the regime of micro-scale inertia (black circles) and its border (dark-grey circles). Note that the numerical datum (square) of Bosse et al. (2005b) is slightly larger than the lower branch (dark-grey triangles) but nonetheless provides the correct order of magnitude.

The normalised breakup time $t_{b}^{*}$ is plotted versus $\ell^{*}$ in figure $12(b)$. This figure again shows a clear division between the experimental data lying in the two regimes of macro-scale (dark-grey triangles) and micro-scale (black circle) inertia. For $\ell^{*} \gtrsim 1000$, the Oseenlet simulations tend towards the Stokeslet simulations and $t_{b}^{*}$ increases with $N_{0}$, as previously found by Metzger et al. (2007). Good agreement is obtained with the experimental data belonging to the Stokes regime (asterisks and light-grey triangles; note that the data labelled with the light-grey triangles result from two different sets of experiments: a set corresponds to $\ell^{*} \approx 300$ with $N_{0} \approx 650$ and another to $\ell^{*} \gtrsim 1000$ but with $N_{0} \approx 2900$ and $R e_{c} \approx 0.1$, thus lying just at the border between the Stokes and macro-scale-inertia regimes). As $\ell^{*}$ is decreased, $t_{b}^{*}$ becomes independent of $N_{0}$. The numerical results gather on a single curve presenting a decrease with decreasing $\ell^{*}$. Excellent quantitative agreement is found with the experimental data belonging to the regime of micro-scale inertia (black circles) and its border (dark-grey circles).

\section{Conclusions}

By performing experimental investigations as well as numerical simulations, we have examined the dynamics of clouds of particles at small but finite Reynolds number. While at zero Reynolds number the dynamics of falling clouds of particles is 
governed only by the initial number of particles $\left(N_{0}\right)$, the dynamics of falling clouds at small but finite Reynolds number (with $S t \ll 1$ ) is governed by two additional dimensionless parameters, namely the cloud Reynolds number $\left(R e_{c}\right)$ and the inertial length normalised by the initial radius of the cloud $\left(\ell^{*}=\ell / R_{0}\right)$.

We identified different regimes: a Stokes regime for low $R e_{c}<0.1$ and large $\ell^{*}>10$ which splits into two inertial regimes in which macro-scale inertia and micro-scale inertia become successively dominant. If the Reynolds number at the cloud scale, $R e_{c}$, is large $\left(R e_{c}>0.1\right)$ but the inertial length is still large $\left(\ell^{*}>10\right)$, macro-scale inertia is dominant, while if $\ell^{*}<10$, micro-scale inertia prevails, and the cloud is driven by far-field Oseen interactions.

In both inertial regimes, the cloud is seen to flatten and to transition into a torus which further widens and eventually breaks up into droplets. While this evolution resembles that observed in the Stokes regime, the physical mechanisms involved are qualitatively different. Whereas in the Stokes regime the evolution towards a torus shape is due to particle depletion near the vertical axis caused by particle leakage, here in the inertial regimes it is due to fluid inflow at the rear of the cloud. A consequence is that particle leakage diminishes with increasing inertia. The breakup processes also differ. Whereas in the Stokes regime the breakup occurs for a critical aspect ratio for which the front-incoming streamlines are able to poke through its centre, it happens at a larger aspect ratio in the inertial regime because the front-incoming flow has to overcome the rear-incoming flow.

As inertia is increased, the evolution of the cloud deformation is accelerated, and the dependence on the initial number of particles within the cloud, $N_{0}$, becomes weaker. This general evolution is found to be qualitatively similar in regimes of both macro- and micro-scale inertia. However, quantitative differences are observed. This is particularly in evidence in the variation of the breakup time with $R e_{c}$ or $\ell^{*}$, where one can clearly identify a division between the data corresponding to the regimes of macro- and micro-scale inertia. The experiments are in good agreement with the simulations of Bosse et al. $(2005 a, b)$ in the macro-scale-inertia regime and with the Oseenlet simulations inspired by Subramanian \& Koch (2008) in the microscale-inertia regime.

Supplementary movies are available at journals.cambridge.org/flm.

\section{REFERENCES}

Adachi, K., Kiriyama, S. \& Koshioka, N. 1978 The behaviour of a swarm of particles moving in a viscous fluid. Chem. Engng Sci. 33, 115-121.

Bosse, T., Favre, J., Meiburg, E. \& Kleiser, L. 2005 a Settling and breakup of suspension drops. Award-winning video entry to the Gallery of Fluid Motion, 57th APS/DFD Annual Meeting, Nov. 21-23, 2004, Seattle, WA, USA. Phys. Fluids 17, 091107.

Bosse, T., Kleiser, L., Härtel, C. \& Meiburg, E. $2005 b$ Numerical simulation of finite Reynolds number suspension drops settling under gravity. Phys. Fluids 17, 037101.

Bush, J. W. M., Thurber, B. A., \& Blanchette F. 2003 Particle clouds in homogeneous and stratified environments. J. Fluid Mech. 489, 29-54.

Ekiel-Jeżewska, M. L., Metzger, B. \& Guazzelli, É. 2006 Spherical cloud of point particles falling in a viscous fluid. Phys. Fluids 18, 038104.

Lamb, H. 1932 Fluid Mechanics, 6th edn. Dover.

Machu, G., Meile, W., Nitsche, L. C. \& Schaflinger, U. 2001 Coalescence, torus formation and breakup of sedimenting drops: experiments and computer simulations. J. Fluid Mech. 447, 299-336. 
Metzger, B., Nicolas, M. \& Guazzelli, É. 2007 Falling clouds of particles in viscous fluids. J. Fluid Mech. 580, 283-301.

Nitsche, J. M. \& BAtCheloR, G. K. 1997 Break-up of a falling drop containing dispersed particles. J. Fluid Mech. 340, 161-175.

Noh, Y. \& Fernando, H. J. S. 1993 The transition in the sedimentation pattern of a particle cloud. Phys. Fluids A 5, 3049-3055.

Park, J., Metzger, B., Guazzelli, É. \& Butler, J. E. 2010 A cloud of rigid fibres sedimenting in a viscous fluid. J. Fluid Mech. 648, 351-362.

Subramanian, G. \& Koch, D. L. 2008 Evolution of clusters of sedimenting low-Reynolds-number particles with Oseen interactions. J. Fluid Mech. 603, 63-100. 